\title{
Erratum: Consistency of the Adiabatic Theorem \\ [Phys. Rev. Lett. 102, 220401 (2009)]
}

M. H. S. Amin

(Received 5 June 2015; published 14 July 2015)

DOI: 10.1103/PhysRevLett.115.039902

PACS numbers: 03.65.Ta, 03.65.Ca, 03.67.Lx, 99.10.Cd

Equation (6) in the Letter should read

$$
E_{0,1}=\mp \frac{1}{2} \Omega, \quad\left|E_{0,1}\right\rangle=\left(\begin{array}{c}
\alpha^{ \pm} \\
\pm \operatorname{sgn}\left[\sin \left(\omega_{0} t\right)\right] \alpha^{\mp}
\end{array}\right) .
$$

This does not affect any other equations and the conclusion of the paper.

We thank Dr. Dafa Li for bringing this to our attention. 